\title{
Electronic structure and lifetime of an excimer state of HeF
}

\author{
I. D. Petsalakis \\ Theoretical and Physical Chemistry Institute, National Hellenic Research Foundation, 48, Vas. \\ Constantinou Ave., Athens 11635 , Greece
}

(Received 17 July 1990; accepted 16 October 1990)

\begin{abstract}
Theoretical calculations have been carried out on the few lowest electronic states of $\mathrm{HeF}$ in an effort to identify a possible excimer state. The results show that the $2^{2} \Sigma+$ state is bound with a minimum at $2.0 \mathrm{bohr}$. The calculated predissociation rates of the $v=0, N=1$ level of the $2^{2} \Sigma^{+}$state are lower by two orders of magnitude than the rate of the radiative transition $2^{2} \Sigma^{+}$, which has a vertical transition energy of $9.4 \mathrm{eV}$.
\end{abstract}

\section{INTRODUCTION}

The observation of laser emission in rare gas halides, $\mathrm{XeBr}, \mathrm{KrF}, \mathrm{XeCl}$, and $\mathrm{XeF}$, has made the excimer states of these systems interesting to experimentalists and theoreticians. ${ }^{1-3}$ A simple model of an ion-pair state $\mathrm{Rg}^{+} \mathrm{X}^{-}$has been employed for the description of the excimer state in these systems. $A b$ initio calculations on $\mathrm{KrF}^{2}$ essentially confirmed the ion-pair character of the excited state involved in the observed transitions. In addition, the above calculations obtained a crossing at short internuclear distances of the ionpair state with a Rydberg state correlating with $\mathrm{Rg}^{*}+\mathrm{X} . A b$ initio calculations on $\mathrm{ArF}^{*}$ and $\mathrm{XeF}$ (referred to by $\mathrm{Krauss}^{3}$ ) indicate similar electronic structures for the excited states of these systems as in $\mathrm{KrF}$. There have not been any $a b$ initio calculations on the excimer states of $\mathrm{HeF}$, while $\mathrm{NeF}$ has been calculated along with $\mathrm{ArF}, \mathrm{KrF}$, and $\mathrm{XeF}$ by Dunning and Hay. ${ }^{2}$ However it might be expected that the Rydberg states would gain importance in $\mathrm{HeF}$ as the ionization potential of $\mathrm{He}$ is very high. In particular, the lower dissociation limits would involve $\mathrm{He}+\mathrm{F}^{*}$, since the limits $\mathrm{He}^{*}+\mathrm{F}$ lie above the ionization limit of the fluorine atom. ${ }^{4}$ Hocker ${ }^{5}$ has invoked a bound and a dissociating state of $\mathrm{HeF}$ in a two-state model for the creation of the atomic fluorine laser. It is thus of interest to calculate the electronic states of $\mathrm{HeF}$. It might be noted that $\mathrm{HeF}$ being isoelectronic to the Rydberg molecule NeH, also falls into the category of Rydberg molecules although it is expected that the electronic structure of $\mathrm{HeF}^{*}$ will not be as simple as that of $\mathrm{NeH}^{*}{ }^{7}$

In the present work theoretical calculations have been carried out on the first few electronic states of HeF, four of ${ }^{2} \Sigma^{+}$. symmetry and three ${ }^{2} \Pi$, along with the possible coupling between them which might lead to predissociation processes of the bound states.

\section{CALCULATIONS}

The MRD-CI method was used ${ }^{8,9}$ for the electronic calculations while for the vibrational-rotational analysis an analytic method was used ${ }^{10,11}$ involving real basis functions. The AO basis set employed for the electronic calculations consisted of the $(a+b)$ basis set of Theodorakopoulos et $a l .^{12}$ for He and for $\mathrm{F}$ the basis set of Dunning and Hay including the suggested polarization and diffuse functions. ${ }^{13}$ The ground state MO are used as the one-electron basis set for the CI calculations. Reference sets of 34 and 25 configurations with selection thresholds of 10 and $5 \mu$ hartrees were used for the ${ }^{2} \Sigma^{+}$and ${ }^{2} I I$ electronic states, respectively. This type of configuration selection gave for small internuclear distances $(R), C I$ spaces of 15000 configurations while for larger this number decreases smoothly and goes to 5000 for $R$ larger than 3.0 bohr.

\section{RESULTS AND DISCUSSION}

All the calculated potential energy curves are given in Fig. 1 for values of $R$ from 1.0 to 5.0 bohr. The first two electronic states, $1^{2} \Sigma^{+}$and $1^{2} \Pi$, correlating with ground state atoms, are repulsive for the internuclear distances cal-

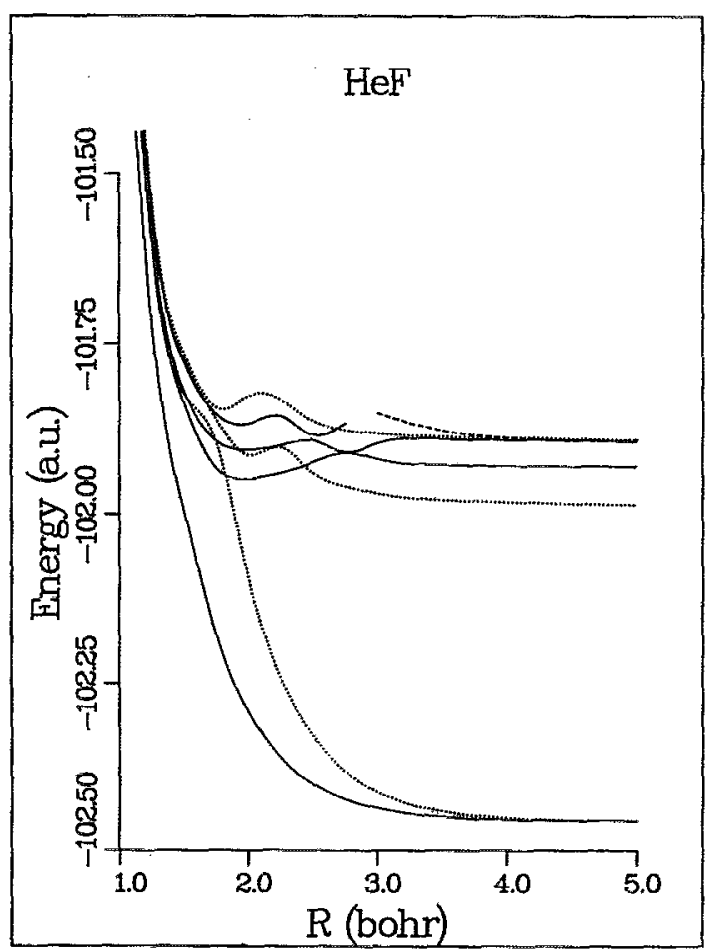

FIG. 1. Potential energy curves of the four lowest ${ }^{2} \Sigma^{+}$electronic states (solid lines) the three lowest ${ }^{2} \mathrm{II}$ (dotted lines) of HeF. The dashed line at $R>3.0$ bohr corresponds to a ${ }^{2} \Delta$ electronic state. 
culated (see Fig. 1). The possible existence of van der Waals minima on these curves has not been of interest in the present work. The $1^{2} \Pi$ state shows a shoulder at about $1.75 \mathrm{bohr}$, where an avoided crossing is found with the higher ${ }^{2} \Pi$ state. The $2^{2} \Pi$ and $3^{2} \Pi$ states also show maxima which might indicate other avoided crossing with higher-lying ${ }^{2} \Pi$ states, not calculated here.

The excited ${ }^{2} \Sigma^{+}$states show minima at $R$ of about 2.0 bohr at vertical transition energies of $9.36,10.51$, and 11.58 $\mathrm{eV}$ for the $2^{2} \Sigma^{+}, 3^{2} \Sigma^{+}$, and $4{ }^{2} \Sigma^{+}$states, respectively, with respect to the $1^{2} \Sigma^{+}$state. At larger $R$ the potential energy curves show features indicating a series of avoided crossing of bound states with a repulsive state. Thus the twostate model of Hocker ${ }^{5}$ needs to be extended to include a series of bound states interacting with the repulsive state. These features in the potential energy curves are reflected in the configurations which are important for each state at different $R$, given in Tables $I$ and II for the ${ }^{2} \Sigma^{+}$and ${ }^{2} \Pi$ states, respectively.

The minima of the potential energy curves at short $R$ are suggestive of Rydberg states. However in this case, they do not correspond to the equilibrium geometry of the $\mathrm{HeF}^{+}$ion where the calculated minima lie at 3.86 and $4.96 \mathrm{bohr}$ for the ${ }^{3} \Sigma$ and the ${ }^{3} \mathrm{II}$ states, respectively, of $\mathrm{HeF}^{+}+{ }^{14}$

The first excited state ${ }^{2} \Sigma^{+}$, shows a broad minimum at $2.0 \mathrm{bohr}$ and could serve as a candidate for laser emission in $\mathrm{HeF}$. It is thus of interest to calculate the predissociation lifetime vs the radiative lifetime for this state. In Fig. 2, a close-up of the excited states is given where the vibrational levels of $2^{2} \Sigma^{+}$have been also drawn as horizontal lines. The calculated spectroscopic contants of $2^{2} \Sigma+$ of $\mathrm{HeF}$ are $B_{e}=4.48 \mathrm{~cm}^{-1}, \omega_{e}=1745 \mathrm{~cm}^{-1}$, and $\omega_{e} x_{e}=35 \mathrm{~cm}^{-1}$. As shown in Figs. 1 and 2, predissociation of the lowest two vibrational levels of the $2^{2} \Sigma^{+}$state might occur by radial coupling to the ground state and by rotational-electronic coupling to the $1^{2} \Pi$ state. The relevant matrix elements, $\vartheta / \vartheta R$ for radial coupling and RXP for rotational-electronic coupling over the electronic wave functions have been calculated and are plotted as functions of the internuclear distance in Figs. 3 and 4.

TABLE I. The configurations $s^{n}$ that characterize the first four ${ }^{2} \Sigma{ }^{+}$states of $\mathrm{HeF}$ at each geometry from 1.0 to 5.0 bohr.

\begin{tabular}{|c|c|c|c|c|}
\hline$R$ & $1^{2} \Sigma^{+}$ & $2^{2} \Sigma+$ & $3^{2} \Sigma^{+}$ & $4^{2} \Sigma t$ \\
\hline 1.0 & $1 \pi_{x}^{2} 1 \pi_{y}^{2} 4 \sigma$ & $1 \pi_{x}^{2} 1 \pi_{y}^{2} 5 \sigma$ & $1 \pi_{x}^{2} 1 \pi_{y}^{2} 6 \sigma$ & $1 \pi_{x}^{2} 1 \pi_{y}^{2} 7 \sigma$ \\
\hline 2.25 & $"$ & $"$ & $"$ & $1 \pi_{y}^{2} 4 \sigma 1 \pi_{x} 5 \pi_{x}$ \\
\hline 2.5 & $"$ & $"$ & $1 \pi_{y}^{2} 4 \sigma 1 \pi_{x} 5 \pi_{x}$ & $1 \pi_{x}^{2} 1 \pi_{y}^{2} 6 \sigma$ \\
\hline 2.75 & $"$ & "t & $"$ & $"$ \\
\hline 3.0 & $"$ & $1 \pi_{y}^{2} 4 \sigma 1 \pi_{x} 5 \pi_{x}$ & $1 \pi_{x}^{2} 1 \pi_{y}^{2} 5 \sigma$ & \\
\hline 3.5 & $"$ & " & $\begin{array}{l}1 \pi_{x}^{2} 1 \pi_{y}^{2} 5 \sigma \\
4 \sigma^{2} 1 \pi_{y}^{2} 5 \sigma \\
4 \sigma^{2} 1 \pi_{x}^{2} 5 \sigma\end{array}$ & \\
\hline 4.0 & $"$ & $"$ & $"$ & \\
\hline 4.5 & $"$ & $"$ & $"$ & \\
\hline 5.0 & $"$ & $"$ & $"$ & \\
\hline
\end{tabular}

"All the above configurations have the first six electrons occupied at the $1 \sigma^{2} 2 \sigma^{2} 3 \sigma^{2}$ core.
TABLE II. The configurations ${ }^{\mathrm{n}}$ that characterize the first three ${ }^{2} \Pi$ states of $\mathrm{HeF}$ at each geometry from 1.0 to 5.0 bohr.

\begin{tabular}{|c|c|c|c|}
\hline$R$ & $1^{2} \Pi$ & $2{ }^{2} \pi$ & $3^{2} \Pi$ \\
\hline 1.0 & $\begin{array}{l}1 \pi_{x}^{2} 1 \pi_{y}^{2} 5 \pi_{x} \\
1 \pi_{x}^{2} 1 \pi_{y}^{2} 3 \pi_{x}\end{array}$ & $1 \pi_{x}^{2} 1 \pi_{y}^{2} 4 \pi_{x}$ & $\begin{array}{l}1 \pi_{x}^{2} 1 \pi_{y}^{2} 3 \pi_{x} \\
1 \pi_{x}^{2} 1 \pi_{y}^{2} 5 \pi_{x} \\
1 \pi_{x}^{2} 1 \pi_{y}^{2} 2 \pi_{x} \\
{ }_{y}\end{array}$ \\
\hline 1.75 & $\begin{array}{l}4 \sigma^{2} 1 \pi_{y}^{2} 1 \pi_{x} \\
\|\end{array}$ & $\begin{array}{l}1 \pi_{x}^{2} 1 \pi_{y}^{2} 5 \pi_{x} \\
1 \pi_{x}^{2} 1 \pi_{y}^{3} 3 \pi_{x} \\
\end{array}$ & $\begin{array}{l}1 \pi_{x}^{2} 1 \pi_{y}^{2} 4 \pi_{x} \\
1 \pi_{x}^{2} 1 \pi_{y}^{2} 5 \pi_{x}\end{array}$ \\
\hline 2.0 & " & $1 \pi_{y}^{2} 4 \sigma 5 \sigma 1 \pi_{x}$ & $\begin{array}{l}1 \pi_{x}^{2} 1 \pi_{y}^{2} 5 \pi_{x} \\
1 \pi_{x}^{2} 1 \pi_{y}^{2} 3 \pi_{x}\end{array}$ \\
\hline 2.25 & $"$ & $"$ & $1 \pi_{y}^{2} 4 \sigma 5 \sigma 1 \pi_{x}$ \\
\hline 3.0 & $"$ & $"$ & $"$ \\
\hline 4.0 & $"$ & $"$ & $"$ \\
\hline 5.0 & $"$ & $"$ & " \\
\hline
\end{tabular}

"All the above configurations have the first six electrons occupied at the $1 \sigma^{2} 2 \sigma^{2} 3 \sigma^{2}$ core

The simultaneous presence of the various coupling interactions would be best accomodated in a multichannel treatment of predissociation. This shall be the subject of future work. For the present, we shall follow the practice ${ }^{11,15}$ of treating each channel separately. The method employed has been described elsewhere. ${ }^{10.11}$ The linewidths are calculated with the aid of the Golden Rule formula

$$
\Gamma=2 \pi\left|\left\langle\varphi_{\nu}|\mathrm{OP}(R)| X_{i}\right\rangle\right|^{2},
$$

where $\varphi_{v}$ and $X_{i}$ stand for the bound and the continuum

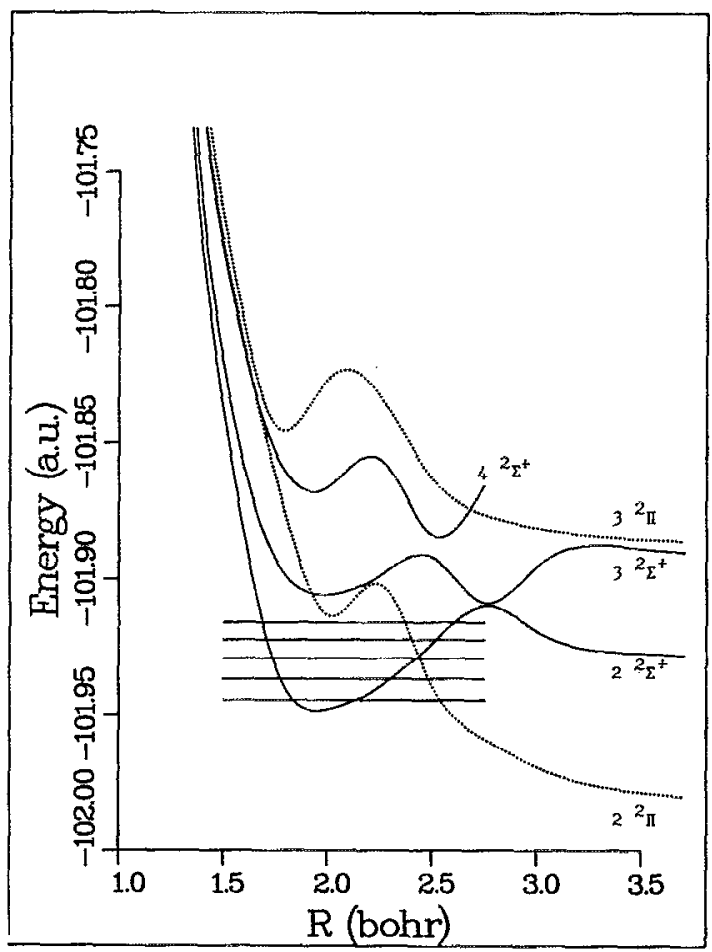

FIG. 2. A close up of the potential energy curves of the excited states in the short $R$ region. The vibrational levels of $2^{2} \Sigma^{+}$are indicated by horizontal lines. 


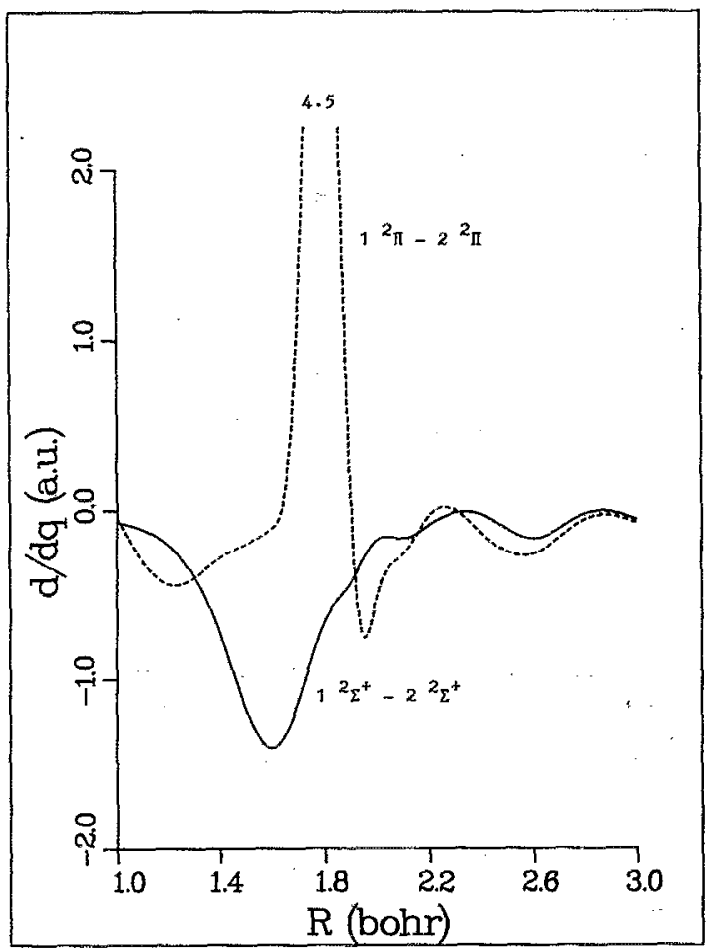

FIG. 3. Radial coupling matrix element $(\vartheta / \vartheta R)$ between the $1^{2} \Sigma^{+}-2^{2} \Sigma^{+}$(solid line) and $1^{2} \Pi-2^{2} \Pi$ (dotted line) electronic states of HeF.

wave functions, respectively, at energy $\varepsilon_{v}$. In the present work the operator $\mathrm{OP}(R)$ for radial coupling is given by

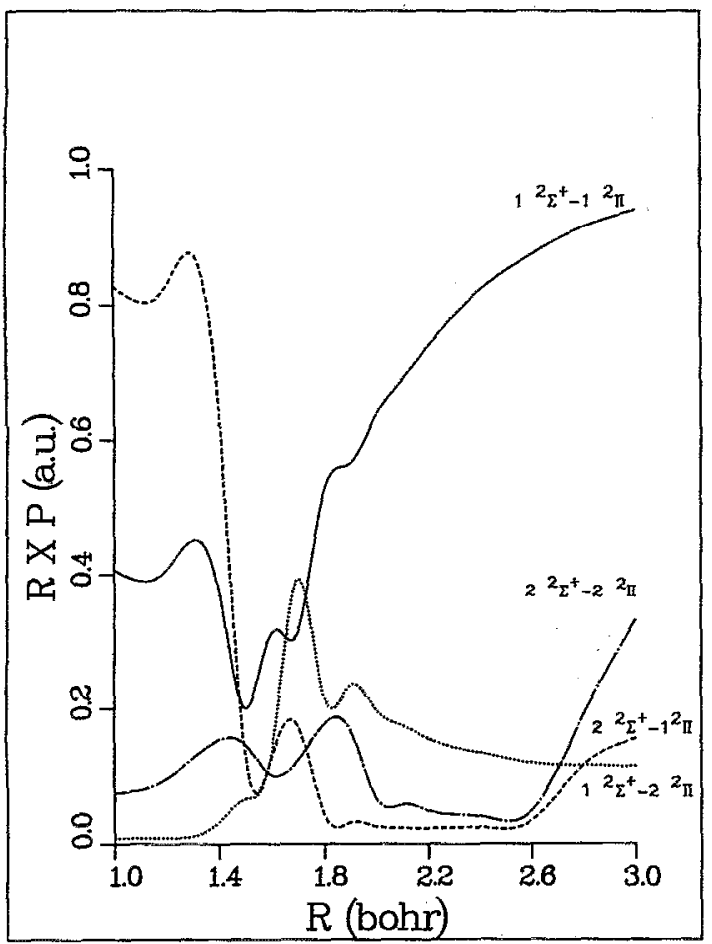

FIG. 4. Rotational-electronic coupling matrix elements for the $1^{2} \Sigma^{+}-1^{2} \Pi$ (solid line), $1^{2} \Sigma^{+}-2^{2} \Pi$ (dotted line), $2^{2} \Sigma^{+}-1{ }^{2} \Pi$ (dashed line), and $2^{2} \Sigma^{+}-2^{2} \Pi$ (dotted-dashed line) pairs of states.
TABLE III. Transition rates $\left(\mathrm{s}^{-1}\right)$ for the radiative and nonradiative transitions from the second ${ }^{2} \Sigma^{+}$state to the lower $1^{2} \Sigma^{+}$and $1^{2} \Pi$ continuum states.

\begin{tabular}{ccc}
\hline & $2^{2} \Sigma^{+}-1^{2} \Sigma^{+}$ & $2^{2} \Sigma^{+}-1^{2} \Pi$ \\
\hline$K_{\text {rad }}$ & $0.12 \times 10^{8}$ & $0.97 \times 10^{6}$ \\
$K_{\text {pre }}$ & $0.5^{\mathrm{a}} \times 10^{6}$ & $0.3^{\mathrm{b}} \times 10^{6}$
\end{tabular}

${ }^{a}$ Using the vibrational coupling operator [Eq. (2)].

${ }^{b}$ Using the rotational coupling operator [Eq. (3)].

$$
\mathrm{OP}(R)=-\frac{\hbar^{2}}{\mu} A(R) \frac{\vartheta}{\vartheta R},
$$

where $A(R)$ is an analytic fit to the electronic matrix elements of $\vartheta / \vartheta R$. by

For rotational-electronic coupling the operator is given

$\mathrm{OP}(R)=-\frac{\hbar}{2 \mu} \sqrt{(N \pm \Lambda)(N+1 \bar{\mp} \Lambda)}\left\langle\frac{\Psi_{i}^{\mathrm{el}}|L \pm| \Psi_{j}^{\mathrm{el}}}{R^{2}}\right\rangle$

where $N=1$ and $\Lambda$ refer to the predissociating state.

The rate of a radiative transition from a bound to a dissociating electronic state is given by

$$
A_{v}=\int_{0}^{E} A(E) d E
$$

where

$$
A(E)=\frac{64 \pi^{4} v^{3}}{3 h}\left|\left\langle\varphi_{v}|D(R)| X_{i}\right\rangle\right|^{2}
$$

and $D(R)$ is an analytic fit to the electronic matrix elements of the dipole transition moment.

The calculated rates of predissociation of the $v=0$, $N=1$ level of the $2^{2} \Sigma^{+}$state by $1^{2} \Sigma^{+}$and by $1^{2} \Pi$ as well as the rates of the radiative transition $2^{2} \Sigma^{+}-1^{2} \Sigma^{+}$and $2^{2} \Sigma^{+}-1^{2} \Pi$ are given in Table III. As shown in Table III, the radiative transition $2^{2} \Sigma+-1^{2} \Sigma^{+}$has the highest probability. The predissociation rates are lower for $v=0, N=1$ level by about two powers of ten. Thus the radiative process would expected to remain important, even if a more accurate treatment of the predissociation, were to be employed, such as a multichannel treatment as well as the possible inclusion of the second derivative $\vartheta^{2} / \vartheta R^{2}$ in the radial coupling operator. On the basis of these results (see Table III) the lifetime of the $v=0, N=1$ level of ${ }^{2} \Sigma+$ is estimated as $83 \mathrm{~ns}$, which is essentially the radiative lifelime. Of course rotational predissociation of the state will gain importance for higher $N$ levels [cf. Eq. (3)]. For $N=10$, the predissociation rate due to the $2^{2} \Sigma^{+}-1^{2} \Pi$ interaction and the radiative rate will be about equal.

In conclusion, the present calculations have shown that the $2^{2} \Sigma^{+}$state of $\mathrm{HeF}$ is bound and has a high radiative transition probability to the ground state, with a transition energy of $9.4 \mathrm{eV}$. The higher lying ${ }^{2} \Sigma^{+}$states also show mimima at short $R$. However it is expected that nonradiative 
processes will be very important in these states as may be seen from the potential energy curves (see Fig. 2).

'J. J. Ewing and C. A. Brau, Phys. Rev. A 12, 129 (1975); J. E. Velazco and D. W. Setser, J. Chem. Phys. 62, 1990 (1975); M. F. Colde and B. A. Thrush, Chem. Phys. Lett. 29, 486 (1974).

${ }^{2}$ T. H. Dunning, Jr. and P. J. Hay, Appl. Phys. Lett. 28, 649 (1976); J. Chem. Phys. 69, 134 (1978).

"M. Krauss, J. Chem. Phys. 67, 1712 (1977).

${ }^{4}$ C. E. Moore, Atomic Energy Levels, Natl. Bur. Stand. Circ. No 467 (U.S. GPO, Washington, D.C., 1949).

"L. O. Hocker, J. Opt. Soc. Am. 68, 262 (1978).

'G. Herzberg, Annu. Rev. Phys. Chem. 38, 27 (1987).

'G. Theodorakopoulos, I. D. Petsalakis, and R. J. Buenker, J. Phys. B 20, 5335 (1987).

${ }^{8}$ R. J. Buenker and S. D. Peyerimhoff, Theor. Chim. Acta 35, 33 (1974); 39, 219 (1975); R. J. Buenker, S. D. Peyerimhoff, and W. Butscher, Mol.
Phys. 35, 771 (1978).

${ }^{9}$ R. J. Buenker, in Studies in Physical and Theoretical Chemistry. Current Aspects of Quantum Chemistry, 1981, edited by R. Carbo (Elsevier, Amsterdam, 1982), Vol. 21, p. 17; R. J. Buenker and R. A. Philips, J. Mol. Struct. 123, 291 (1985).

${ }^{10}$ I. D. Petsalakis; G. Theodorakopoulos, and R. J. Buenker, Phys. Rev. A 38, 4004 (1988).

"I. D. Petsalakis, G. Theodorakopoulos, and R. J. Buenker, J. Chem. Phys. 92, 4920 (1990).

${ }^{12} \mathrm{G}$. Theodorakopoulos, S. C. Farantos, R. J. Buenker, and S. D. Peyerimhoff, J. Phys. B 17, 1453 (1984).

${ }^{13}$ T. H. Dunning Jr. and P. J. Hay, in Methods of Electronic Structure Theo$r y$, editẹd by H. F. Shaefer III (Plenum, New York, 1977).

${ }^{14}$ P. W. Harland, R. G. A. R. Maclagan, and R. W. Simpson, J. Chem. Soc. Faraday Trans. 2, 84, 1847 (1988).

${ }^{15}$ M. M. Graff, J. T. Moseley, J. Durup, and E. Rouett, J. Chem. Phys. 78, 2355. (1983). 